\title{
Applications of Optical Coherence Tomography for Nondestructive Evaluation of Materials
}

\author{
Jim Slepicka ${ }^{1}$
}

${ }^{1}$ Baxter Healthcare Corporation, Round Lake, IL USA

Of the various tomographic imaging methods that have become commonplace such as confocal microscopy, computed tomography (CT), and magnetic resonance imaging (MRI), Optical Coherence Tomography (OCT) has perhaps undergone the most rapid technical development, finding use in an ever-widening range of applications. While relatively new, OCT is filling a long sought-after gap in depth-resolved optical imaging techniques. While its use over the past 25 years has been primarily as a diagnostic tool for ophthalmology and pathology, its role in nondestructive evaluation (NDE) of materials and structures is gaining wider acceptance as new approaches and novel uses are explored. This is due to its relative simplicity, high resolution and speed, non-contact/non-destructive nature, and flexibility in application, not to mention affordability. Some typical applications include inspection of composites and multi-layer films, characterization of coatings and particles, surface profilometry, and visualization of two-phase flows.

OCT is somewhat akin to ultrasound imaging in that backscattered energy from an incident source is analyzed to form depth information. In fact, OCT cross-sections bear a striking resemblance to ultrasound images except that the lateral and axial resolution is increased by at least an order of magnitude. This is because OCT utilizes low coherence interferometric principles to interrogate various depths within the sample, with signal parameters based on the wavelength of light- typically in the near infrared region- compared to the much longer acoustic wavelengths used in ultrasound imaging. The earliest OCT schemes operated in time-domain (TD-OCT). This involves splitting the source light into two beams with one directed at the sample and the other directed to an oscillating reference mirror which provides a changing optical pathlength. The reference light is then mixed with the light continuously reflecting back from different depths within the specimen. Constructive interference occurs when the pathlengths of light reflected by various scattering sites within the depth of the sample match the reference beam pathlength at any given moment, within an envelope whose width is related to the coherence length of the source. Thus, if the source is of low coherence, i.e., broadband such as an LED, the interference signal from a single scatterer will only occur when the two paths are virtually equal- typically within a few microns. Further information regarding operation theory including newer frequency-domain systems can be found in Reference 1.

While penetration depth is typically limited by signal loss due to multiple scattering in optically dense materials, its use with transparent/translucent materials can reveal features deeper than is possible from traditional tissue scanning. For example, this is advantageous for inspection of through-transmission laser welds (TTLW, clear part welded to absorbing substrate), identification/localization of particulate matter in polymer materials, or characterization of sealing interfaces such as tubing bonded to various fittings. Following are examples from some recent investigations pertaining to material characterization or inspection.

Figure 1 shows a cross-section of a $0.75 \mathrm{~mm}$ thick multi-layer polymer film heat sealed to a rigid injection-molded rib. Three distinct features are observed: (1) the three layers of the film are evident in the unsealed portion of the film on the left, (2) the diminished thickness of the sealed portion of the film 
(top right) with the softened material pushed to the left (bulge), and (3) the sealing process has changed the shape of the normally rectangular rib (bottom left).

Generation of a B-scan (2D image as shown above) requires a single lateral scan of the specimen beam across the sample as depth information is processed for each position resulting in the columns of pixels for the image. Going one step further, a stack of B-scans (C scan) can be obtained by rastering lateral scans. This produces a 3D data set analogous to those produced by confocal microscopy or CT/MRI but where the stack consists of vertical cross-sections. Figure 2 shows a rendered image from a C-scan of high-density particulate matter embedded in silicone rubber. Shadow under particle is an indication of the higher optical density of the material.

\section{References:}

[1] A F Fercher et al, Reports on Progress in Physics, Volume 66, Number 2, 2003.
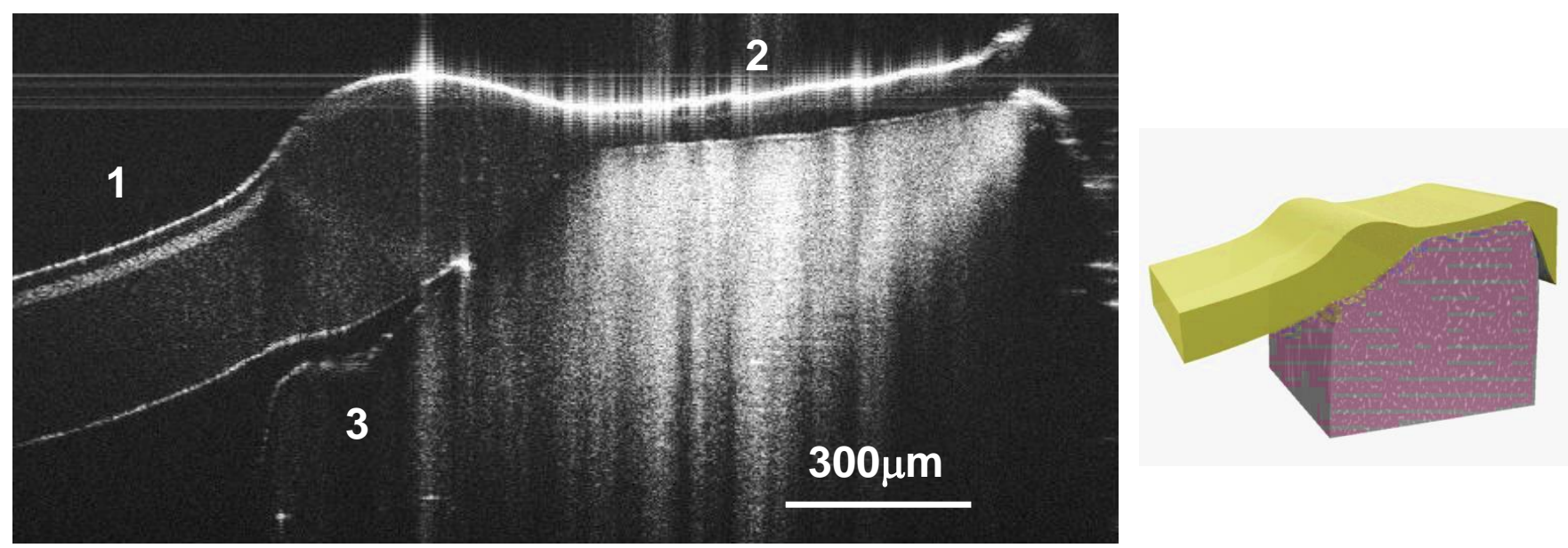

Figure 1. Multilayer polymer film heat sealed to rigid rib (cutaway diagram on right).

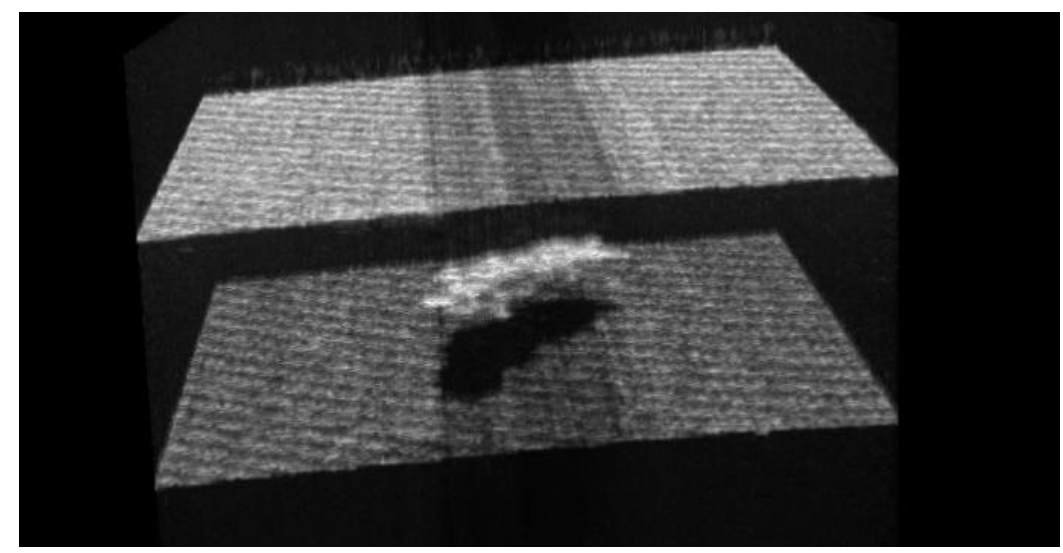

Figure 2. Particulate matter embedded in silicone rubber. 